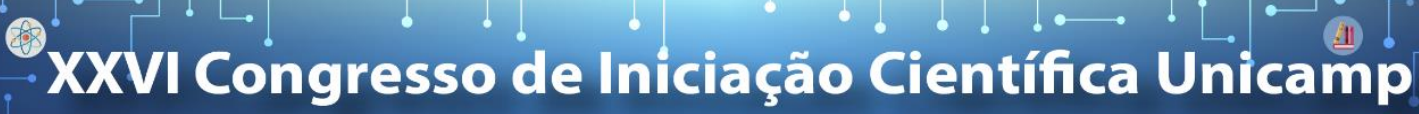

\section{Study of Tools for Similarity Matching of Concept Maps}

\section{Tales Abdo dos Santos*, Gisele Busichia Baioco}

\begin{abstract}
Concept maps are very useful for representing knowledge and their use has been extended to various areas. However, tasks that need concept maps comparison require a lot of effort from the analyst and are subject to interpretation loss. This project had as objetive identify and characterize tools to compare concept maps by similarity. Therefore, it was studied the principles that envolve concept maps construction and similarity tools and methods that help to search and visualize similar elements in conceps maps.
\end{abstract}

\section{Key words: \\ Similarity Matching, Concept Maps Similarity, Concept Maps Matching}

\section{Introduction}

Concept maps are graphical elements that show the connections between the concepts that make up a particular domain of knowledge, as [1] Novak and Cañas declare.

Given their nature, they make easier to understand and analyze explicit knowledge. However, activities involving the comparison of concept maps may require great effort on the part of the analyst.

Concept maps may be considered as a complex data type, since they are composed of a structure that relates concepts. Therefore, it is necessary to search and analyze comparison by similarity tools of complex data, whose structure is similar to concept maps structure, in order to be possible the effective comparative analysis of concept maps.

\section{Results and Discussion}

In order to find articles related to the research, two searches were made with principles of systematic review to find articles containing algorithms with metrics for concept maps matching. The first search did not bring articles as expected; most of them were about graphs, which could serve, but would not bring good results. The second focused only on concept maps matching, which brought more suitable articles for the research. Besides that, in both searches were also included articles from other sources, such as other people indications and recommendations of the article bases.

Having promising articles to form a good base in the research, the authors of each article were contacted. Many of them answered, however unfortunately it was not possible to obtain any of the algorithms used by them. Therefore, three promising articles, well developed and with the necessary information for the research, were analyzed.

The articles bring methods to be followed that adjust the values needed for the metric used. Each one applies to a particular problem, which demonstrates that this area of research is embracing. Among them, the one that stood out was the article by [2] Chen, Lin and Chang. They make modifications on a metric made by [3] Goldsmith to achieve the purpose of the search. Attributes are added in the concepts and links to find the most important points of each concept map of the comparison, thus generating a more refined result and considering more than just the map structures.

Tools were also found, with interfaces, that brought much to the research. These tools had unique features such as a nice layout, easy use, or good application of the chosen metric. As with the articles, one tool stood out: [4] CMapTools, developed by the Florida Institute of Human Cognition and Machinery.

CMapTools has proved very good in several aspects, including its metric, interface and use. The only problem with the tool is its low data volume. It is possible to perform only a simultaneous comparison, but this does not make it unfeasible.

There are implementations for maps matching in specific, however mostly are not disclosed or have been discontinued. There are some that are open for anyone, on platforms as GitHub, but they don't have support in its use or aren't well explained.

\section{Conclusions}

As much as the use of concept maps has been increasing a lot, the efficient and automated matching of these has not grown as fast, which exposed the lack that open source implementations do in this area. Many of the articles and tools found have been made in the past decade, while the articles seen in this decade mostly did not bring anything concrete to this research, besides reinforcing the demand of concept maps matching solutions.

\section{Acknowledgement}

I thank Franciene Duarte and Juan Galindo for all the help and assistance at the beginning of the research and $\mathrm{PIBIC/CNPq}$ for all the assistance during these months.

\footnotetext{
${ }^{1}$ NOVAK, J. D.; CAÑAS, A. J. The theory underlying concept maps and how to construct and use them. 2008.

${ }^{2}$ CHEN, S. -W; LIN, S. C.; CHANG, K. E. Attributed Concept Maps: Fuzzy Integration and Fuzzy Matching. 2001, 31, 5.

${ }^{3}$ GOLDSMITH, T. E.; JOHNSON, P. J.; ACTON, W. H. Assessing structural knowledge. 1991, 83, 88.

${ }^{4}$ CAÑAS, A. J.; HILL, G.; CARFF, R.; SURI, N.; LOTT, J.; GÓMEZ, G.; ESKRIDGE, T.; ARROYO, M.; CARVAJAL, R. CmapTools: A Knowledge Modeling and Sharing Environment. 2004.
} 